\title{
Delayed Diagnosis of Pulmonary Thromboembolism Due to Overfocus on COVID-19
}

\author{
Yukinori Harada, Taro Shimizu \\ Department of Diagnostic and Generalist Medicine, Dokkyo Medical University Hospital, Mibu, Tochigi, Japan
}

Received: 15/09/2020

Accepted: 20/09/2020

Published: $21 / 10 / 2020$

How to cite this article: Harada Y, Shimizu T. Delayed diagnosis of pulmonary thromboembolism due to overfocus on COVID-19. EJCRIM 2020;7: doi:10.12890/2020_002002.

Conflicts of Interests: The Authors declare that there are no competing interests.

This article is licensed under a Commons Attribution Non-Commercial 4.0 License

\section{ABSTRACT}

The coronavirus disease 2019 (COVID-19) pandemic has made it difficult for physicians to follow their usual diagnostic processes. We present the case of a 25-year-old man with adjustment disorder who developed dyspnoea. He was concerned about COVID-19, but his test result was negative. After excluding COVID-19, the physician concluded that his symptoms were related to his psychiatric condition. However, the patient was diagnosed with pulmonary thromboembolism by another physician. To avoid missing a diagnosis, physicians must practice zero-based thinking, regardless of COVID-19 concerns, and not be distracted from the patient's core problems.

\section{LEARNING POINTS}

- $\quad$ During the COVID-19 pandemic, significant diagnostic errors can occur because physicians are so concerned about COVID-19 that they may ignore other diagnoses.

- To avoid missing a life-threatening condition during the COVID-19 pandemic, physicians must consider zero-based thinking, regardless of COVID-19 concerns, and not be distracted from the patient's core problems.

- Measuring vital signs after a short walk can be helpful for investigating suspected pulmonary thromboembolism.

\section{KEYWORDS}

COVID-19, diagnostic errors, pandemic, pulmonary thromboembolism

\section{CASE DESCRIPTION}

A 25-year-old man was referred to the diagnostic and generalist outpatient department for evaluation of a 1-month history of dyspnoea. He had frequently experienced recurrent nephrotic syndrome due to minimal change disease since he was 14 years old and had been repeatedly treated, mainly with prednisolone. He also regularly visited a psychiatrist because of adjustment disorder with depressive mood for a year. One month previously, he had developed an ongoing cough, dyspnoea and low-grade fever of $37.5^{\circ}$. He also developed a sore throat and nasal discharge 2 weeks later. He visited the hospital concerned about coronavirus disease 2019 (COVID-19), but his test result was negative. Because his symptoms disappeared at rest and his oxygen saturation was $97 \%$ on ambient air, his respiratory symptoms were not regarded as serious or as requiring intervention. Meanwhile, significant proteinuria was detected, which prompted a diagnosis of recurrent nephrotic syndrome and he was restarted on oral prednisolone. During the following 10 days, his dyspnoea worsened, and he developed bilateral leg oedema and experienced two episodes of syncope. However, because oxygen saturation in the examination room was not low enough to possibly cause dyspnoea, the physician, the patient and his mother all thought that his symptoms were related to his psychiatric condition. However, 7 days previously, because his psychiatrist was concerned about other causes of his dyspnoeic symptoms, he had been referred to the diagnostic and generalist outpatient department. He noted palpitations on exertion, but did not have chest pain, haemoptysis, or leg 
swelling or pain. He had never smoked, and had no family history of any coagulation disorders. He denied recent long-distance travel, trauma or surgery.

On examination, he was slightly tachypnoeic with a respiratory rate of 22 breaths per min. There was also significant tachycardia with a pulse rate of $133 \mathrm{bpm}$ in the sitting position, which reduced to under $100 \mathrm{bpm}$ in the supine position. His blood pressure was $105 / 75 \mathrm{mmHg}$, body temperature was $36.7^{\circ}$, and oxygen saturation was $97 \%$ on ambient air. There was no jugular venous distention, heart murmur or pulmonary rales. Slight slow-pitting oedema was noted on both legs, but there was no erythema or tenderness.

\section{Methods and Procedures}

Based on his low-grade fever, tachycardia and dyspnoea, high-output cardiac failure due to thyrotoxicosis was initially suspected, but his thyrotropin level was normal, and there was no enlargement of the cardiac silhouette. Other laboratory data were normal apart from a low serum albumin level $(2.4 \mathrm{~g} / \mathrm{dl})$ and a slightly increased spot urine protein level (100 mg/dl). The electrocardiogram showed sinus tachycardia with a heart rate of 110 bpm, small Q waves on II, III and aVF leads, and negative T waves on V1 and V2 leads, which was not thought to be suggestive.

At the time, because the patient admitted that he felt palpitations and dyspnoea while standing and walking but not in the supine position, orthostatic dysregulation and platypnea-orthodeoxia syndrome were considered. The orthostatic test detected an increase of $40 \mathrm{bpm}$ in heart rate with mild dyspnoea, but there was no change in blood pressure or oxygen saturation. This finding was consistent with postural orthostatic tachycardia syndrome, which can cause mild dyspnoea. However, when the patient walked on the spot for about 1 minute, his oxygen saturation dropped to $92 \%$ and his dyspnoea worsened. Pulmonary thromboembolism was considered given that nephrotic syndrome has a known high risk of pulmonary thromboembolism. Computed tomography angiography revealed multiple thrombi in the bilateral pulmonary artery (Fig. 1) and the right common ileac vein. The patient was admitted with the diagnosis of pulmonary and deep venous thromboembolism. He started a 1-week course of intravenous unfractionated heparin, followed by oral apixaban and was discharged without any residual symptoms.

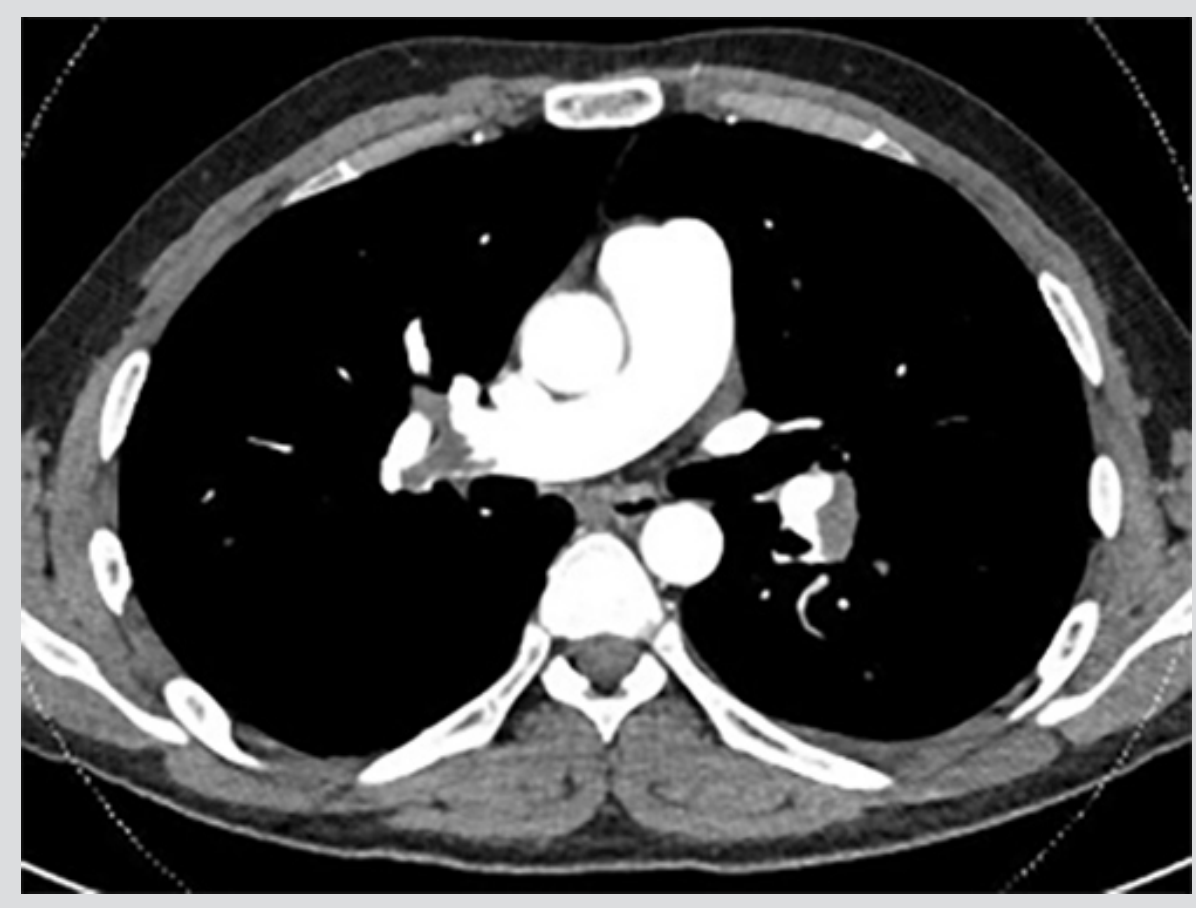

Figure 1. Computed tomography angiography showing thrombi in the bilateral pulmonary arteries

\section{DISCUSSION}

This case reminds us that the COVID-19 pandemic has made it difficult for physicians to follow the correct diagnostic process. In this case, both the physician and the patient were deeply concerned about COVID-19 as the number of positive COVID-19 cases was rising in Japan. In addition, although the patient complained of dyspnoea, his oxygen saturation at rest was normal. These factors and the fact that he had a mental illness, which could contribute to symptoms being overlooked during a pandemic ${ }^{[1]}$, may have led the physician to reject other causes 


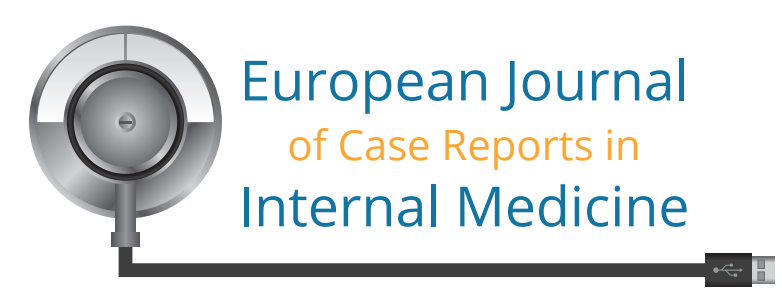

of dyspnoea after ruling out COVID-19 in this patient. As demonstrated in this case, we should be cautious that the cognitive process is not distorted during the pandemic ${ }^{[1,2]}$. To avoid failure to diagnose life-threatening conditions, physicians must consider zero-based thinking, regardless of COVID-19 concerns, without being distracted from the patient's core problem ${ }^{[3]}$.

If a pulmonary thromboembolism is suspected, a short walk can be an easy and helpful option. A previous study reported that in patients without chronic pulmonary disease who terminated the 6-minute walking test early because oxygen saturation had dropped below $90 \%$, one third had a pulmonary thromboembolism ${ }^{[4]}$. In our case, although the vital signs were within the normal range at rest, standing and walking induced dramatic changes in heart rate and oxygen saturation, which led the physicians to consider a life-threatening condition such as pulmonary thromboembolism. To avoid missing a diagnosis, physicians should measure vital signs after a short walk in patients complaining of dyspnoea but with normal oxygen saturation at rest.

\section{REFERENCES}

1. Boyle JG, Walters MR, Jamieson S, Durning SJ. Clinical reasoning in the wild: premature closure during the COVID-19 pandemic. Diagnosis (Berl) 2020;7:177-179.

2. Schiff G, Mirica M. COVID-19: making the right diagnosis. Diagnosis (Berl) 2020 Jul 13;/j/dx.ahead-of-print/dx-2020-0063/dx-2020-0063.xml. doi: 10.1515/dx-2020-0063. Online ahead of print.

3. Yokose M, Harada Y, Shimizu T. The reply. Am J Med 2020;133:e328.

4. Fuglebjerg NJU, Jensen TO, Hoyer N, Ryrsø CK, Madsen BL, Harboe ZB. Silent hypoxia in patients with SARS CoV-2 infection before hospital discharge. Int J Infect Dis 2020;99:100-101. 